\title{
Retracted: Green Chemistry Approach for Efficient Synthesis of Schiff Bases of Isatin Derivatives and Evaluation of Their Antibacterial Activities
}

\author{
Journal of Nanoparticles \\ Received 16 December 2015; Accepted 16 December 2015 \\ Copyright (C) 2015 Journal of Nanoparticles. This is an open access article distributed under the Creative Commons Attribution \\ License, which permits unrestricted use, distribution, and reproduction in any medium, provided the original work is properly \\ cited.
}

The paper titled "Green Chemistry Approach for Efficient Synthesis of Schiff Bases of Isatin Derivatives and Evaluation of Their Antibacterial Activities" [1], published in Journal of Nanoparticles, has been retracted as it was accepted for publication on the basis of peer review reports that were submitted from fraudulent reviewer accounts.

In late 2014, a number of publishers discovered widespread abuse of the peer review process, including cases of identity theft and faked review reports. In July 2015, Hindawi concluded an extensive investigation into peer review fraud and identified a number of articles that had been accepted on the basis of fraudulent peer review reports. In accordance with the recommendations of the Committee on Publication Ethics (COPE), Hindawi sent these manuscripts for re-review using independent Editorial Board Members. Following this re-review process, this article has been retracted as it was deemed unsuitable for publication.

\section{References}

[1] J. Panda, V. J. Patro, B. M. Sahoo, and J. Mishra, "Green chemistry approach for efficient synthesis of schiff bases of isatin derivatives and evaluation of their antibacterial activities," Journal of Nanoparticles, vol. 2013, Article ID 549502, 5 pages, 2013. 


\author{
Jnyanaranjan Panda, ${ }^{1}$ V. Jagannath Patro, ${ }^{1}$ Biswa Mohan Sahoo, ${ }^{1}$ and Jitendriya Mishra ${ }^{2}$ \\ ${ }^{1}$ Department of Pharmaceutical Chemistry, Roland Institute of Pharmaceutical Sciences and Berhampur, Odisha 760010, India \\ ${ }^{2}$ University Institute of Pharmaceutical Sciences, UGC Centre of Advanced Study (CAS), Panjab University, Chandigarh 160014, India
}

Correspondence should be addressed to Jnyanaranjan Panda; jnyana@rediffmail.com

Received 31 December 2012; Accepted 12 January 2013

Academic Editor: Amir Kajbafvala

Copyright (C 2013 Jnyanaranjan Panda et al. This is an open access article distributed under the Creative Commons Attribution License, which permits unrestricted use, distribution, and reproduction in any medium, provided the original work is properly cited.

\begin{abstract}
Microwave-assisted organic synthesis, a green chemistry approach, is nowadays widely used in the drug synthesis. Microwaveassisted synthesis improves both throughput and turnaround time for medicinal chemists by offering the benefits of drastically reduced reaction times, increased yields, and pure products. Schiff bases are the important class of organic compounds due to their flexibility, and structural diversities due to the presence of azomethine group which is helpful for elucidating the mechanism of transformation and rasemination reaction in biological system. This novel compound could also act as valuable ligands for the development of new chemical entities. In the present work, some Schiff bases of Isatin derivatives was synthesized using microwave heating method. Schiff base of Isatin were synthesized by condensation of the keto group of Isatin with different aromatic primary amines. They were characterized by means of spectral data and subsequently subjected to the in vitro antibacterial activities against gram positive and gram negative strains of microbes. It was observed that the compound with electron withdrawing substituents exhibited good antibacterial activities against almost all the micro organisms.
\end{abstract}

\section{Introduction}

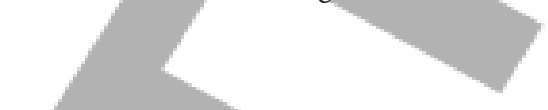

Microwave-assisted organic synthesis is widely used as a source of heating in drug synthesis. Drug molecules can be built in a fraction of the time by this method. As a result, this technique has rapidly gained acceptance as a valuable tool for accelerating drug discovery and development processes. A microwave is a form of electromagnetic energy, which falls at the lower end of the electromagnetic spectrum and is defined in a measurement of frequency as 300 to 300,000 Megahertz. The microwave region of the electromagnetic spectrum lies between infrared and radio frequencies. The basic mechanism of microwave assisted synthesis involves agitation of polar molecules or ions that oscillate under the effect of an oscillating electric or magnetic field. In the presence of an oscillating field, particles try to orient themselves or be in phase with the field. Only materials that absorb microwave radiation are relevant to microwave chemistry. These materials can be categorized according to the three main mechanisms of heating such as dipolar polarization, conduction mechanism, and interfacial polarization. The technique offers simple, clean, fast, efficient, and economical for the synthesis of a large number of drug molecules, having provided the momentum for many medicinal chemists to switch from traditional heating method to microwave heating method [1,2]. Thus it follows green chemistry approach. The role of green chemistry is essential in ensuring that the next generation of chemicals, materials, and energy is more sustainable than the current generation. Worldwide demand for environmentally friendly chemical processes and products requires the development of novel and cost-effective approaches to prevent pollution of the environment [3]. The important area of green chemistry is the elimination of solvents during chemical processes or the replacement 
TABLE 1: Comparative study on yield and reaction time of the synthesized compounds $2(\mathrm{a}-\mathrm{j})$ by conventional and microwave assisted method.

\begin{tabular}{|c|c|c|c|c|}
\hline \multirow{2}{*}{ Compound code } & \multicolumn{4}{|c|}{ Conventional method } \\
\hline & Time (h) & \multicolumn{2}{|c|}{ Energy $\left(\right.$ Temp. $\left.{ }^{\circ} \mathrm{C}\right)$} & Yie \\
\hline $2 a$ & 3 & \multicolumn{2}{|c|}{$98-100$} & \\
\hline $2 \mathrm{~b}$ & 3 & \multicolumn{2}{|r|}{$98-100$} & \\
\hline $2 c$ & 3 & \multicolumn{2}{|r|}{$98-100$} & \\
\hline $2 \mathrm{~d}$ & 2 & \multicolumn{2}{|r|}{$98-100$} & \\
\hline $2 \mathrm{e}$ & 2 & \multicolumn{2}{|r|}{$98-100$} & \\
\hline $2 \mathrm{f}$ & 2 & \multicolumn{2}{|r|}{$98-100$} & \\
\hline $2 g$ & 2 & \multicolumn{2}{|r|}{$98-100$} & \\
\hline $2 \mathrm{~h}$ & 3 & \multicolumn{2}{|r|}{$98-100$} & \\
\hline $2 \mathrm{i}$ & 3 & \multicolumn{2}{|r|}{$98-100$} & \\
\hline $2 \mathrm{j}$ & 3 & \multicolumn{2}{|r|}{$98-100$} & \\
\hline \multicolumn{5}{|c|}{$\begin{array}{l}\text { TABLE 2: TLC report and melting point data of the synthesized } \\
\text { compounds } 2(a-j) \text {. }\end{array}$} \\
\hline Compound code & $\mathrm{R}$ & & $\operatorname{Tm}\left({ }^{\circ} \mathrm{C}\right)$ & $R_{f}$ \\
\hline $2 \mathrm{a}$ & phen & & $144-145$ & 0.56 \\
\hline $2 \mathrm{~b}$ & 2-nitrop & & $120-121$ & 0.67 \\
\hline $2 c$ & 3-nitrop & & $140-141$ & 0.62 \\
\hline $2 \mathrm{~d}$ & 4-nitrop & & $150-151$ & 0.68 \\
\hline $2 \mathrm{e}$ & 3-chloro & & $135-136$ & 0.66 \\
\hline $2 \mathrm{f}$ & 4-chloro & & $140-141$ & 0.67 \\
\hline $2 \mathrm{~g}$ & 4-bromo & & $230-231$ & 0.62 \\
\hline $2 \mathrm{~h}$ & 4-fluorol & & $141-142$ & 0.56 \\
\hline $2 \mathrm{i}$ & $3-\mathrm{Cl}-4-\mathrm{F}-$ & nyl & $129-130$ & 0.54 \\
\hline $2 \mathrm{j}$ & 2,6-dichlor & enyl & 241-142 & 0.55 \\
\hline
\end{tabular}

of hazardous solvents with environmental friendly solvents. Heterocyclic compounds are the most commonly used pharmacophore in the development of drugs and pharmaceutical substances. Due to their drug-likeness and structural diversity, these are routinely employed in high-throughput screening at early stages of drug discovery processes. Therefore, the goal of the present study is to carry out the synthesis of some heterocyclic compounds under green chemistry approach [4]. The above facts prompted us to synthesize some Schiff base of Isatin derivatives by using microwave heating method [5-7]. Azomethine group (-C=N-) containing compounds typically known as Schiff bases has been synthesized by the condensation of primary amines with active carbonyls [8]. Schiff bases form a significant class of compounds in medicinal chemistry due to various biological activities such as antibacterial [9], antifungal [10], antiviral [11], anti-HIV [12], and anticonvulsant [13] activities. The structures of the synthesized compounds were confirmed by means of their physical and spectral data. The synthesized compounds were evaluated for their possible antibacterial activities by cup plate method.

\section{Experimental Method}

2.1. Materials. The chemicals and solvents used for the experimental work were of commercial grade. All the melting

\begin{tabular}{|c|c|c|c|}
\hline $\mathrm{d}(\%)$ & Time (min.) & $\begin{array}{c}\text { crowave assisted method } \\
\text { Energy (power. watt) }\end{array}$ & Yield (\%) \\
\hline 64 & 5 & 210 & 84 \\
\hline 62 & 7 & 210 & 82 \\
\hline 56 & 6 & 210 & 85 \\
\hline 58 & 10 & 210 & 75 \\
\hline 66 & 7 & 210 & 85 \\
\hline 53 & 9 & 210 & 74 \\
\hline 57 & 8 & 210 & 77 \\
\hline 53 & 10 & 210 & 76 \\
\hline 56 & 10 & 210 & 72 \\
\hline 60 & 8 & 210 & 84 \\
\hline
\end{tabular}

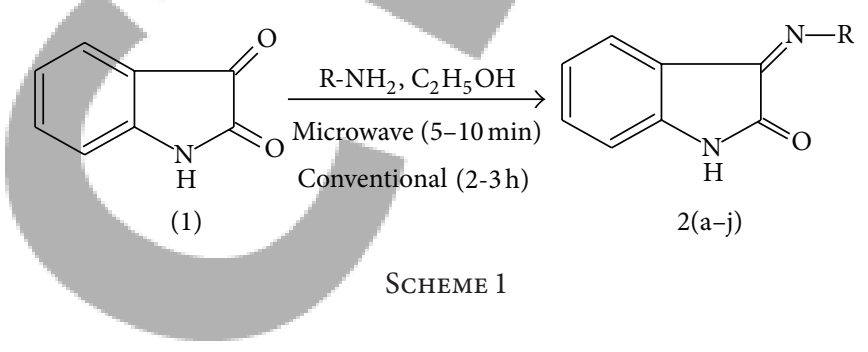

points were taken in open capillaries and are uncorrected (Table 2). Followup of the reactions and checking the purity of the compounds were made by TLC on precoated Silica gel-aluminum plates (Type 60 F254, Merck, Darmstadt, Germany) and were visualized by exposure to UV-light $(254 \mathrm{~nm})$ or iodine vapor for few seconds. The IR spectra of the compounds were recorded on FT-IR Spectrophotometer, model IR Affinity-1 (SHIMADZU), using KBr powder and the values are expressed in $\mathrm{cm}^{-1} .{ }^{1} \mathrm{H}$ NMR spectra of selected compounds were recorded on multinuclear FT NMR Spectrometer, model Advance-II (Bruker), (at $400 \mathrm{MHz}$ ) using tetramethylsilane as an internal standard. The multiplicities of the signals are denoted with the symbols $s, \mathrm{~d}, \mathrm{t}$, and $\mathrm{m}$ for singlet, doublet, triplet, and multiplet, respectively. The microwave irradiated synthesis was performed in scientific microwave oven, Catalyst System (operating between 140$700 \mathrm{~W}$ ). All the reactions were carried out at power level-1, which corresponds to $140 \mathrm{~W}$.

2.2. Conventional Synthesis of Schiff Bases of Isatin. Equimolar $(0.01 \mathrm{~mol})$ quantity of Isatin and substituted anilines were dissolved in ethanol $(10 \mathrm{~mL})$ and refluxed for $3 \mathrm{~h}$ in presence of glacial acetic acid. In between TLC was checked to confirm the completion of reaction. After completion of reaction, the reaction mixture was kept overnight to get the solid product. The product was filtered, dried, and recrystallized from ethanol (Scheme 1).

2.3. Microwave Synthesis of Schiff Bases of Isatin. The required quantities of above reactants are subjected to microwave irradiation for 5-10 min at power level 1 (140 watts). In between TLC was checked to confirm the completion of 
TABLE 3: Antibacterial activities of the synthesized compounds 2(a-j).

\begin{tabular}{|c|c|c|c|c|c|}
\hline \multirow{2}{*}{ Compounds } & \multicolumn{5}{|c|}{ Diameter of zone of inhibition (millimeters) } \\
\hline & P. vulgaris & P. aeruginosa & E. coli & S. aureus & S. epidermidis \\
\hline $2 \mathrm{a}$ & $12.23 \pm 0.57$ & $15.02 \pm 1.00$ & $12.00 \pm 1.00$ & $13.32 \pm 0.57$ & $12.56 \pm 0.57$ \\
\hline $2 b$ & $16.33 \pm 0.55$ & $17.10 \pm 1.00$ & $22.00 \pm 1.00$ & $23.00 \pm 1.00$ & $15.33 \pm 0.57$ \\
\hline $2 c$ & $18.33 \pm 0.55$ & $18.11 \pm 1.00$ & $21.00 \pm 1.00$ & $19.00 \pm 1.00$ & $14.00 \pm 1.00$ \\
\hline $2 \mathrm{~d}$ & $18.66 \pm 0.52$ & $16.00 \pm 1.00$ & $18.66 \pm 0.57$ & $19.34 \pm 0.57$ & $17.00 \pm 1.00$ \\
\hline $2 e$ & $15.33 \pm 0.57$ & $17.00 \pm 1.00$ & $16.33 \pm 0.57$ & $16.32 \pm 0.57$ & $14.33 \pm 0.57$ \\
\hline $2 \mathrm{f}$ & $21.32 \pm 0.53$ & $21.00 \pm 1.00$ & $21.00 \pm 1.00$ & $19.34 \pm 0.57$ & $15.33 \pm 0.57$ \\
\hline $2 g$ & $17.33 \pm 0.57$ & $18.00 \pm 1.00$ & $20.00 \pm 1.00$ & $19.23 \pm 0.57$ & $12.33 \pm 0.57$ \\
\hline $2 \mathrm{~h}$ & $16.33 \pm 0.56$ & $20.10 \pm 1.00$ & $20.00 \pm 1.00$ & $22.20 \pm 1.00$ & $16.33 \pm 0.57$ \\
\hline $2 \mathrm{i}$ & $12.33 \pm 0.57$ & $14.10 \pm 1.00$ & $12.33 \pm 0.57$ & $16.32 \pm 0.57$ & $11.66 \pm 0.57$ \\
\hline $2 j$ & $14.34 \pm 0.53$ & $14.10 \pm 1.00$ & $14.33 \pm 0.57$ & $17.35 \pm 0.57$ & $16.00 \pm 1.00$ \\
\hline Control & - & - & & & - \\
\hline Standard & $23.33 \pm 0.57$ & $20.33 \pm 0.57$ & $31.33 \pm 0.57$ & $21.66 \pm 0.57$ & $23.33 \pm 0.57$ \\
\hline
\end{tabular}

Results were expressed as mean \pm S.D. $(n=3)$; “-” indicates no zone of inhibition.

reaction. After completion of reaction, the reaction mixture was kept overnight to get the solid product. The product was filtered, dried, and recrystallized from ethanol (Scheme 1).

\section{Results and Discussion}

The synthetic protocol followed was outlined in Scheme 1. The synthesized compounds were characterized by their physical and spectral studies. As compared to conventional heating, microwave heating provides high yield with pure product in less reaction time (Table 1). The IR spectra of all the synthesized compounds were recorded using $\mathrm{KBr}$ (Merck). All the compounds have exhibited -NH stretching in the region of $3400-3200 \mathrm{~cm}^{-1}$. IR spectra of synthesized compounds showed the presence of characteristic absorption peaks around $3000-3100 \mathrm{~cm}^{-1}(\mathrm{C}-\mathrm{H}, \mathrm{Ar}), 2800-2780 \mathrm{~cm}^{-1}$ $(-\mathrm{C}=\mathrm{N}-), \quad 1640-1602 \mathrm{~cm}^{-1} \quad(\mathrm{C}=\mathrm{N}), \quad 1580-1520 \mathrm{~cm}^{-1} \quad(\mathrm{C}=\mathrm{C}$ $\mathrm{Ar})$, and $1346-1330 \mathrm{~cm}^{-1}(\mathrm{C}-\mathrm{N})$. The halogenated derivatives showed the IR absorption bands in the regions and 1400$1000 \mathrm{~cm}^{-1}, 800-600 \mathrm{~cm}^{-1}$, and $600-500 \mathrm{~cm}^{-1}$ which correspond to (C-F str.), (C-Cl str.), (C-Br str.), respectively. The $\mathrm{N}-\mathrm{O}$ stretching vibrations in nitro group occur near $1550-1475 \mathrm{~cm}^{-1}$ (asymmetrical) and $1365-1290 \mathrm{~cm}^{-1}$ (symmetrical), with the band at $1550 \mathrm{~cm}^{-1}$ being the stronger. The ${ }^{1}$ HNMR spectra above synthesized compounds have shown singlet in the region of $\delta 9.35$ to 9.98 corresponding to secondary amino group $(-\mathrm{NH})$. The aromatic protons resonate as multiplet in the region of $\delta 7.03$ to 7.90 . The synthesized compounds were evaluated for their in-vitro antibacterial activities by cup plate method against both gram positive and gram negative bacteria. The results of the study were summarized in Table 3 including the activity of standard. The compound $2 \mathrm{f}$ showed most promising effect whereas compound $2 \mathrm{a}$ and $2 \mathrm{i}$ were found to be least effective against $P$. vulgaris. Similarly the compound $2 \mathrm{f}$ was found to have maximum zone of inhibition and compounds $2 \mathrm{i}$ and $2 j$ were found to have minimum zone of inhibition against $P$. aeruginosa. The compound $2 \mathrm{~b}$ exhibited most promising effect whereas compound $2 \mathrm{a}$ was found to have mild effect against E. coli and S. aureus. The compound $2 \mathrm{~d}$ showed maximum effect against $S$. epidermidis whereas compound $2 \mathrm{i}$ was found to have mild effect against this species. Most of the tested compounds exhibited antibacterial activities against all the test organisms but the activity was less than that of the standard drug tetracycline in this test concentration. Thus taking into account the antibacterial activities of the tested compounds most of the compounds displayed poor activities against S. epidermidis and promising activities against $P$. vulgaris, $P$. aeruginosa, E. coli, and S. aureus.

The photographs of zone of inhibition of standard, control, and some of the tested compounds were given in Figure 1. Figure 1(a) showed the zone of inhibition of the standard drug against $E$. coli whereas there is no zone of inhibition in case of control. Similarly Figure 1(b) displayed the zone of inhibition of the standard drug against $S$. aureus whereas there is no zone of inhibition in case of control. Figure 1(c) showed the zone of inhibition of test compound $2 \mathrm{~h}(\mathrm{~L})$ which was more than that of $2 \mathrm{a}(\mathrm{R})$ against $S$. aureus. Figure 1(d) showed the zone of inhibition of test compounds $2 \mathrm{i}(\mathrm{L})$ and $2 \mathrm{f}(\mathrm{R})$ against $P$. vulgaris. In case of Figure $1(\mathrm{e})$ it was clearly visible that the diameter of zone of inhibition of compound $2 \mathrm{c}$ was more than that of compound $2 \mathrm{~d}$ against $E$. coli. Figure 1(f) showed the diameter of zone of inhibition of compounds $2 \mathrm{c}$ and $2 \mathrm{~d}$ against $P$. aeruginosa.

\section{Biological Evaluation}

4.1. Antibacterial Study. The synthesized compounds were screened in-vitro for their antibacterial activities against Staphylococcus aureus (MTCC-87), Escherichia coli (MTCC40), Staphylococcus epidermidis (MTCC-2639), Pseudomonas aeruginosa (MTCC-424), and Proteus vulgaris (MTCC 426) using cup plate method $[14,15]$. The compounds were tested at $500 \mu \mathrm{g}$ concentration in dimethyl sulphoxide (DMSO), using nutrient agar as the medium. After $24 \mathrm{~h}$ of incubation 


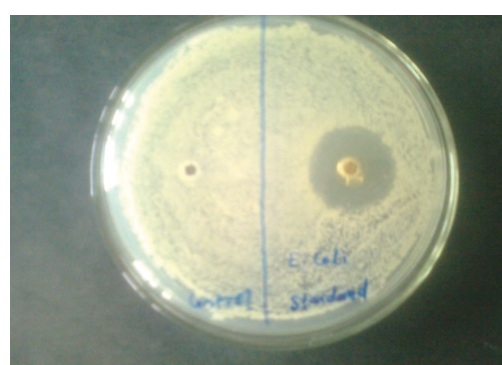

(a) Zone of inhibition of control and standard against $E$. coli

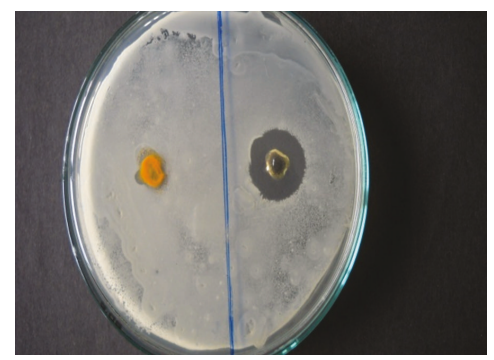

(d) Zone of inhibition of test compounds $2 \mathrm{i}(\mathrm{L})$ and $2 \mathrm{f}(\mathrm{R})$ against $P$. vulgaris

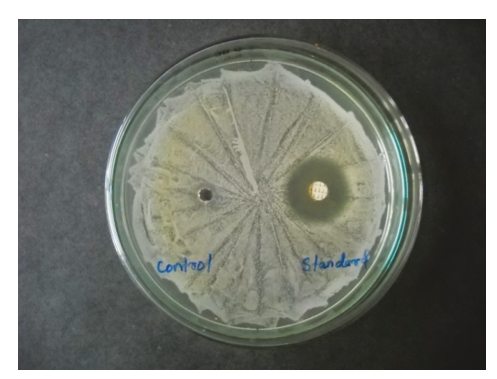

(b) Zone of inhibition of control and standard against S. aureus

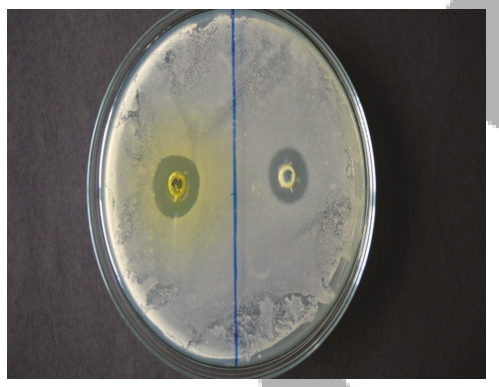

(e) Zone of inhibition of test compounds $2 \mathrm{c}$ (L) and 2d (R) against E. coli

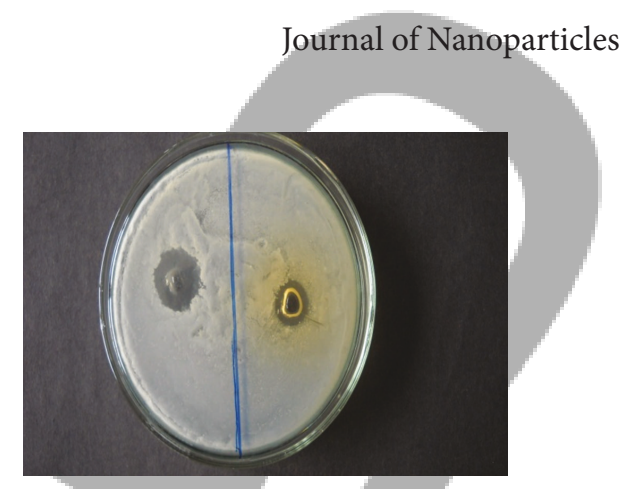

(c) Zone of inhibition of test compounds $2 \mathrm{~h}$ (L) and 2a (R) against S. aureus

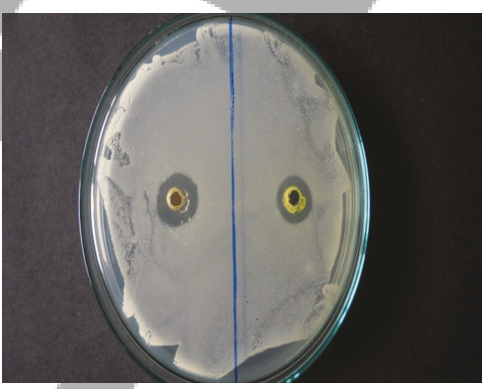

(f) Zone of inhibition of test compounds $2 \mathrm{c}$ $(\mathrm{L})$ and $2 \mathrm{~d}(\mathrm{R})$ against $P$. aeruginosa

FIGURE 1: Photographs displaying zone of inhibition of control, standard, and various test compounds against different test organisms (R: right, L: left).

at $37^{\circ} \mathrm{C}$, the zone of inhibition formed were measured in millimeters against standard drug tetracycline and the data were presented in Table 3.

\section{Conclusion}

We have synthesized some Schiff base of Isatin derivatives by conventional and microwave irradiation method. With the help of microwave synthesis, the yield of product was increased from $60 \%$ upto $85 \%$ as compared to conventional method. By microwave irradiation the reactions were completed within 5-10 minutes and the products were obtained in good to high yields, which reduced the time, waste, and formation of byproduct. The microwave assisted synthesis is simple ecofriendly and can be used as an alternative to the existing conventional heating method. From the results of antibacterial studies it was concluded that the tested compounds exhibited significant antibacterial activities against both gram positive and gram negative organisms. Among the tested compounds, compound substituted with electron withdrawing group in Isatin residue preferably at paraposition showed promising antibacterial activities; this may be attributed to their enhanced electronic character which favors greater penetration through microbial membrane.

\section{Acknowledgments}

The authors are very much thankful to Roland Institute of Pharmaceutical Sciences, Berhampur, Odisha, India, for providing necessary facilities to carry out the research work.
The authors are also thankful to SAIF, Panjab University, Chandigarh, for spectral analysis of synthesized compounds.

\section{References}

[1] M. Charde, A. Shukla, V. Bukhariya, J. Mehta, and R. Chakole, "A review on: a significance of microwave assist technique in green chemistry," International Journal of Phytopharmacy, vol. 2, no. 2, pp. 39-50, 2012.

[2] S. Ravichandran and E. Karthikeyan, "Microwave synthesisa potential tool for green chemistry," International Journal of ChemTech Research, vol. 3, no. 1, pp. 466-470, 2011.

[3] S. Sharma, S. Gangal, and A. Rauf, "Green chemistry approach to the sustainable advancement to the synthesis of heterocyclic chemistry," Rasayan Journal of Chemistry, vol. 1, no. 4, pp. 693717, 2008.

[4] M. Kidwai, "Green chemistry trends toward sustainability," Pure and Applied Chemistry, vol. 78, no. 11, pp. 1983-1992, 2006.

[5] H. M. Mamun, M. A. Foysal, and M. Mahabub, "Microwaveassisted efficient synthesis of isatins and spiro-thiadiazolines under green chemistry protocol," Journal of Scientific Research, vol. 2, no. 2, pp. 322-329, 2010.

[6] A. Kulshrestha and S. Baluja, "Microwave promoted synthesis of some Schiff bases," Archives of Applied Science Research, vol. 2, no. 3, pp. 221-224, 2010.

[7] R. Somani, S. Pawar, S. Nikam, P. Shirodkar, and V. Kadam, "Microwave assisted synthesis and antimicrobial activity of some Schiff's bases," International Journal of ChemTech Research, vol. 2, no. 2, p. 860, 2010.

[8] S. K. Sridhar, S. N. Pandeya, J. P. Stables, and A. Ramesh, "Anticonvulsant activity of hydrazones, Schiff and Mannich 
bases of isatin derivatives," European Journal of Pharmaceutical Sciences, vol. 16, no. 3, pp. 129-132, 2002.

[9] S. N. Pandeya, D. Sriram, G. Nath, and E. De Clercq, "Synthesis and antimicrobial activity of Schiff and Mannich bases of isatin and its derivatives with pyrimidine," Farmaco, vol. 54, no. 9, pp. 624-628, 1999.

[10] A. Jarrahpour, D. Khalili, E. De Clercq, C. Salmi, and J. M. Brunel, "Synthesis, antibacterial, antifungal and antiviral activity evaluation of some new bis-Schiff bases of isatin and their derivatives," Molecules, vol. 12, no. 8, pp. 1720-1730, 2007.

[11] S. S. Chhajed and M. S. Padwal, "Antimicrobial evaluation of some novel Schiff and Mannich bases of isatin and its derivatives with quinolin," International Journal of ChemTech Research, vol. 2, no. 1, pp. 209-213, 2010.

[12] T. R. Bal, B. Anand, P. Yogeeswari, and D. Sriram, "Synthesis and evaluation of anti-HIV activity of isatin $\beta$-thiosemicarbazone derivatives," Bioorganic and Medicinal Chemistry Letters, vol. 15, no. 20, pp. 4451-4455, 2005.

[13] M. Verma, S. N. Pandeya, K. N. Singh, and J. P. Stables, "Anticonvulsant activity of Schiff bases of isatin derivatives," Acta Pharmaceutica, vol. 54, no. 1, pp. 49-56, 2004.

[14] S. H. Gillespie, Medical Microbiology-Illustrated, Butterworth Heinemann, London, UK, 1994.

[15] U. K. Singh, S. N. Pandeya, A. Singh, B. K. Srivastava, and M. Pandey, "Synthesis and antimicrobial activity of Schiff's and NMannich bases of isatin and its derivatives with 4-Amino-Ncarbamimidoyl benzene sulfonamide," International Journal of Pharmaceutical Sciences and Drug Research, vol. 2, no. 2, pp. 151-

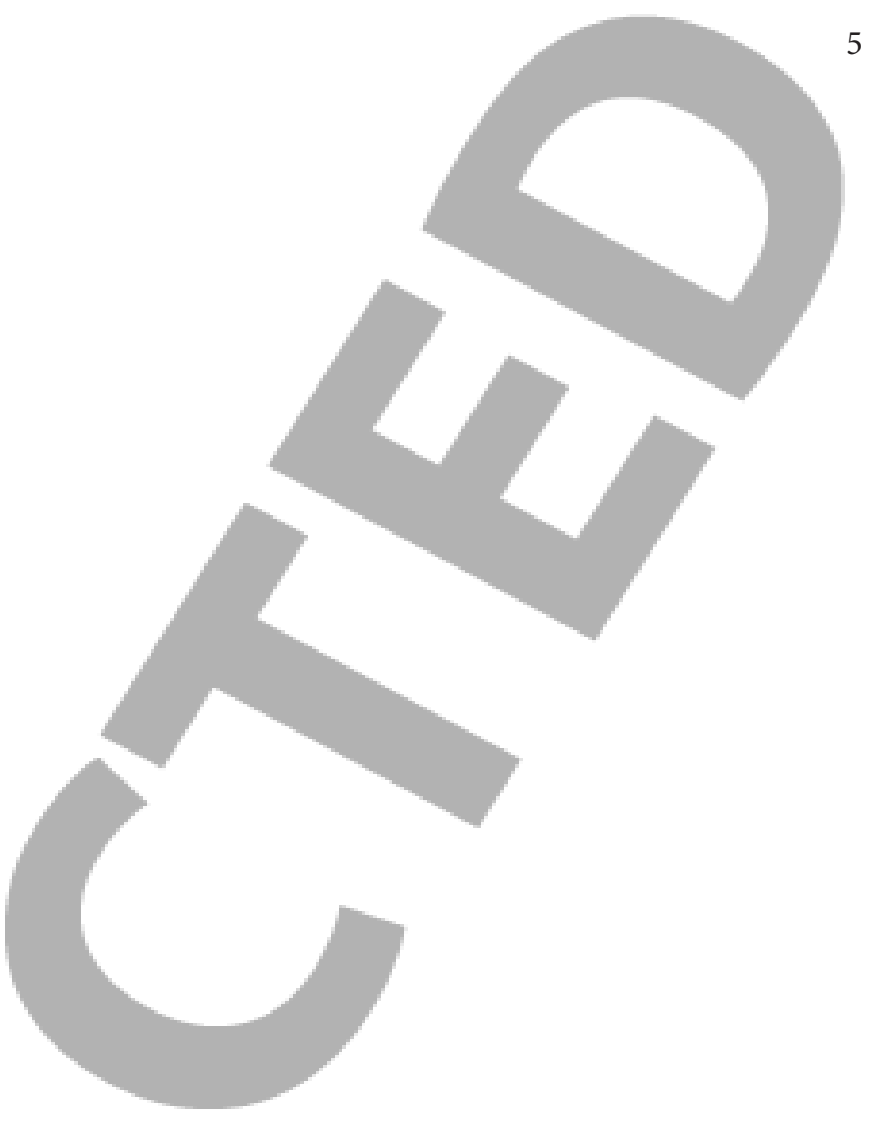
154, 2010 